\title{
PICCOLA BIBLIOTECA
}

Paola Rigo. Memoria classica e memoria biblica in Dante. Firenze: Olschki, 1994 (Saggi di Lettere Italiane). Pp. 183.

Questo saggio di Paola Rigo consegue brillantemente lo scopo che si propone, quello di rendere ragione del rapporto di Dante con gli illustri precedenti delle tradizioni biblica e classica. Pur non avendo pretese di sistematicità - anche perché il lavoro è una silloge di cinque articoli, di cui solo tre pubblicati pre-cedentemente - la studiosa ha il grande merito di aver investigato il campo dei riferimenti biblico-classici con un rigore esemplare fondato sulla capillare consultazione delle fonti. Prova di questo atteggiamento è l'imponenza e la ricchezza delle note bibliografiche sempre corredate di ampi stralci del testo sub iudice.

Ciò che colloca la Rigo nel vivo del più recente dibattito esegetico che riguarda Dante e i classici è la sua piena concordanza con l'idea di privilegiare l'apporto che a Dante derivò dai Commenti e dagli Accessus agli Autori; e l'acutezza dell'autrice consiste nel non essersi limitata ad indagare la sfera dell'esegesi antica dei classici, ma di averla allargata a quella dell'esegesi della Bibbia e in particolare alla liturgia.

Al lavoro non nuoce peraltro un'appropriata organizzazione della materia, che si dipana agli occhi del lettore con una certa 'suspense', per meglio rendere, forse, quella che Montale ha chiamato la "somma enorme di corrispondenze, di richiami che la lettera suscita, rinviandoci ai suoi echi, ai suoi giochi di specchi, alle sue rifrazioni" (53). E forse, come ribadiremo fra breve, più che l'idea di una 'gradazione' o 'accumulazione strategica', il concetto della 'simultaneità' di certi echi è il frutto più invitante del saggio.

Il volume si apre con lo studio La discesa agli Inferi nella Vita Nova, un interessantissimo esame delle possibili fonti del viaggio 'gnoseologico' e allo stesso tempo poetico che Dante traccia nel suo primo scritto di rilievo. Pur nella convinzione che si possa trattar solo di "suggestioni nate da un gioco di concordanze" (20), la studiosa ci conduce attraverso il motivo del "pasto del cuore" (Vita Nova 3.3-7) fino alla nota "scena del gabbo" (cap. 14) rintracciando una lunga serie di riferimenti al contempo scritturali (Ezechiele, Apocalisse) e virgiliani, che indicano come Dante tratti o quantomeno alluda a una discesa agli inferi già in tale contesto. Un elemento di grande interesse è la rilevanza data all'esegesi sia biblica (S. Gerolamo, Glossa Ordinaria, S. Gregorio), sia virgiliana (Bernardo Silvestre) per accertare la rete di riferimento intertestuale. Questo tratto infatti collega l'autrice ai più recenti tentativi di definire il modo in cui Dante leggeva i classici e cioè attraverso l'ineliminabile mediazione dell'esegesi.

Il secondo articolo, Tempo Liturgico nell'Epistola ai Principi e ai popoli d'Italia, riguarda invece l'esatta valutazione degli echi biblici presenti nella famosa epistola dantesca. Questi echi, come la Rigo dimostra, sono organizzati secondo un unico filo conduttore cronologico che va dal periodo dell'Avvento a quello della Pasqua in una serie di riferimenti incentrati specialmente sul Salmo 94 ed il suo rapporto con la liturgia, il capitolo 21 del Vangelo di Luca ed il famoso passo dell'epistola paolina ai Romani concernente la consacrazione e legittimazione divina del potere imperiale. Il 
tutto segue una "linea di transito rappresa tra il sollievo del risveglio e la pena della tenebra" (43), a conferma di una nuova applicazione della 'matrice' o 'modello' dell'Esodo, tanto cara al Singleton, e che "rivive nel rito religioso dell'anno e del giorno" (44). Ciò che più conta infatti è il modo in cui la studiosa è riuscita a dimostrare come in Dante la liturgia sia un potente veicolo della lezione biblica.

Il terzo lungo studio, Tra Maligno e Sanguigno, diviso in quattro sezioni, quasi avendo l'episodio di Paolo e Francesca a pretesto, affronta l'elemento metodologico cardine del saggio della Rigo: la tecnica dantesca della citazione classica e biblica.

Pur senza assumerlo come obbiettivo principale del proprio studio nel passare in rassegna i diversi comportamenti di Dante allorché si confronta con i modelli classici e vetero o neotestamentari, l'autrice mostra un atteggiamento tendenzialmente classificatorio che prevede la sistemazione del materiale a diversi livelli: si va dalla semplice citazione alla 'correzione' o re-interpretazione (fatto, questo, assai ben studiato dalla critica nordamericana), per giungere fino alla 'manipolazione' del modello (emblematico è il caso di Gerione), alla sua 'scomposizione paradigmatica' (la serie di exempla alterni di Purg. 24, 25 e 26) e, inoltre, - e questo è forse il maggior tratto di novità dello studio - all'effetto di 'simultaneità intertestuale' che alcuni luoghi danteschi presentano e che la studiosa definisce con grande chiarezza: "Non si tratta di snidare un senso nascosto, ma di misurare la forza evocativa della lettera poetica che racchiude in sé più e più potenzialità semantiche, tutte simultaneamente presenti" (79). A volte, poi, le immagini della Scrittura hanno lasciato la loro impronta nella fantasia dantesca a causa della loro 'immediata corporeità' e il Poeta le sfrutta proprio in tal senso, 'retrocedendo' la metafora alla sua lettera (Purg. 23.10-12 e 19.73), mediante quello che la Rigo definisce "arresto di citazione" (85).

Lo studio si conclude con una suggestiva interpretazione del verso "tignemmo il mondo di sanguigno", che è posta nella cornice della fisiologia medievale, e perciò tenuto conto del fatto che sanguigno non equivale a 'sangue' (che è per di più un hapax della Commedia) - grazie all'illuminante apporto delle fonti della Scuola Medica Salernitana e al peso semantico del termine-spia benignius ("animal grazioso e benigno" è il modo in cui Francesca si rivolge a Dante) è pressoché impossibile escludere che la condanna definitiva di Paolo e Francesca sia stata loro comminata perché rappresentanti emblematici del temperamento 'sanguigno', al quale la fisiologia medievale assegnava coloro per i quali è più difficile correggere e moderare l'innato 'impeto' amoroso della propria natura.

Il quarto saggio, Consorte degli Dei, è una pregevolissima indagine sugli echi dell'episodio di Glauco (Par. 1.64-72), letto dalla studiosa come un'allusione antifrastica al gesto di Adamo e collocato in una interessantissima relazione con la figura di Marsia. Adamo, dopo il peccato, si vesti di pelli insieme ad Eva e pertanto l'essere "tratto" da una "vagina" equivale in questo contesto a una liberazione; "liberazione dallo schermo della mortalità, dalla pelle dei phantasmata che nasconde l'interiore impronta di Dio" (119). Ma il tratto più interessante dello studio è senza dubbio l'interpretazione della iunctura "consorte degli Dei", interpretazione resa possibile ancora una volta da un riferimento alla liturgia (le preghiere dell'Offertorio), nonché all'esegesi, e che ci mostra l' immagine dell'Incarnazione come chiaro "centro del mistero cristiano e centro anche della intera Cantica" (128), in quanto se è vero che all'ingresso del Paradiso il poeta è sospeso al di là dell'umano e al di qua del divino, è 
pur vero che egli ci suggerisce che il "ridivenire immagine perfetta di Dio è imprescindibile dal raggiungimento della pienezza di umanità" (128).

L'ultimo studio della Rigo, Prenderò il cappello (Par. 25.1-12) è quanto mai suggestivo poiché rintraccia il campo semantico di appartenenza del termine 'cappello' non già nella birretatio accademica ma nella incoronazione poetica, per di più arricchita dal senso del sumere pilleum, il gesto cioè dell'affrancatura degli schiavi nell'antica Roma. La studiosa passa in rassegna tutte le fonti latine classiche sul tema e si sofferma anche a valutare l'idea della manumissio in ecclesiis. Dante bannitus dalla sua stessa città può ricomporre il dissidio, o meglio superare l'ostacolo dell'esilio, per mezzo di questa sorta di cerimonia redentiva: "nello stesso luogo, il battistero, in cui fu costituito persona in ecclesia ora Dante augura di riconfermarsi persona nella città secolare. Firenze, la Firenze agognata della riconciliazione, è allo stesso tempo figura di Roma e della Gerusalemme celeste e si collega a tutte le immagini ed i motivi sia biblici che liturgici o politici" (153). La scelta poetica dell'autore, pertanto, "nasce dalla fede nella tradizione giuridica romana, l'unica capace, per Dante, di ripristinare l'accordo perduto tra civitas terrena e civitas celeste, tra la giustizia umana dunque e la giustizia divina" (153).

\section{ANTONIO ROSSINI}

University of Toronto

Lorenzo de' Medici. The Autobiography of Lorenzo de' Medici the Magnificent: A Commentary on My Sonnets. Translated with an introduction by James Wyatt Cook, together with the text of Comento de' Miei Sonetti, reprinted from the critical edition of Tiziano Zanato [Florence: Olschki, 1991]. Binghamton, N.Y.: Center for Medieval and Early Renaissance Studies, 1995 (Medieval and Renaissance Texts and Studies 129). Pp. 289.

Translation is a humble trade; still, each translator works according to some principle. This reviewer believes that the only way to be faithful to the original is to remake it in natural English (or whatever the target language may be). Mr. Cook claims to follow exactly the same principle, but belies the claim in practice. He would have done better to shield himself behind the principle that the best rendering is an ungainly, literal one, permitting the reader with some knowledge of the original language to follow the syntax of the original text, for his translation of Lorenzo de' Medici's commentary is composed in contorted and unnatural English as he attempts to follow the Italian syntax. (Lorenzo's sonnets, however, are adroitly rendered into English blank verse.) Whenever Mr. Cook feels the need to supply some bit of bridging syntax in English, in order to make explicit a connecting link implict in the Italian, he pointlessly puts it inside square brackets instead of simply writing a sentence that would convey to English readers the same notion conveyed by the original to Italian readers. Italian uses many more passive and reflexive constructions than English does, and every translator has to 